\title{
La distribución espacial de la fractalidad temporal de la precipitación en la España peninsular y su relación con el Î́ndice de Concentración
}

\author{
Óliver Meseguer-Ruiz ${ }^{1}$ oliver.meseguer@ub.edu; Javier Martín-Vide ${ }^{1}$; Jorge Olcina \\ Cantos $^{2}$; Pablo Sarricolea Espinoza ${ }^{1,3}$
}

\begin{abstract}
RESUMEN
La dimensión fractal de la distribución temporal de la precipitación (D) es un indicador de la característica de autosimilitud en la distribución de la lluvia en diferentes intervalos temporales. Si bien en su sentido espacial ha sido ampliamente trabajado anteriormente y está bien definido, la interpretación del concepto de fractalidad aplicado a la distribución temporal es abstracto. Se han calculado los valores de D en 20 observatorios de la España peninsular para el período comportado entre 1997 y 2010 a partir de valores 10-minutales. El comportamiento espacial de la dimensión fractal presenta unos valores máximos en la región norte del área de estudio, siendo muy similares al sur, y unos valores mínimos en el este. El Índice de Concentración (CI) expresa el peso relativo de los días más lluviosos de una serie en el total de la precipitación acumulada de esa serie, y es un indicador de la precipitación que ha sido bien estudiado y aplicado a diversas áreas, cuyo significado climático es bien conocido. Los valores del CI son máximos al este y mínimos en la región más septentrional de la península ibérica. Por el comportamiento espacial, se puede intuir una buena correlación negativa entre D y CI, pero que aumenta al calcular la correlación espacial.
\end{abstract}

Palabras clave: Fractalidad temporal, autosimilitud, precipitación, Índice de Concentración, España peninsular.

\section{The spatial distribution of the temporal fractality of precipitation in peninsular Spain and its relationship with the concentration index}

\begin{abstract}
The fractal dimension of the temporal distribution of precipitation (D) is an indicator of the property of self-similarity in the distribution of rainfall at different time intervals. While its spatial meaning has been widely worked previously and is well defined, the interpretation of fractality applied to the temporal distribution is abstract. The values of $\mathrm{D}$ for 20 observatories with 10 -minutes data in mainland Spain have been calculated for the period from 1997 to 2010. The spatial behavior of the Fractal Dimension presents maximum values in the northern region of the study area, being very similar to the south, and minimum values in the east. The Concentration Index (CI) expresses the relative weight of the rainiest days of a series on the total accumulated rainfall of that series, and is an indicator of rainfall that has been well studied and applied to many areas, and its climatic meaning is well known. CI values are minimal in the northern region of the Iberian Peninsula and maximal in the east. For the spatial behavior, it exists a good negative correlation between D and CI but that increases when calculated as a spatial correlation.
\end{abstract}

Keywords: Temporal fractality, self-similarity, precipitation, concentration index, mainland Spain.

Recibido el 20 de julio de 2014, aceptado el 25 de noviembre de 2014.

Grupo de Climatología, Universidad de Barcelona.

Instituto Interuniversitario de Geografía, Universidad de Alicante.

Departamento de Geografía, Universidad de Chile. 


\section{INTRODUCCIÓN}

La variabilidad de las diferentes componentes atmosféricas es notable a cualquier escala temporal que se considere. En la península ibérica, la variable que muestra una mayor dispersión en sus registros es la precipitación, tanto en las cantidades acumuladas como en el reparto temporal de la misma. Esto, por su ubicación entre el Océano Atlántico y un mar casi interior, el Mediterráneo, y por estar situada en la banda transitoria entre las zonas de los anticiclones subtropicales, al sur, y los vientos del oeste y las borrascas, al norte.

El mar Mediterráneo ocupa una extensa área de aproximadamente 2,5 millones de kilómetros cuadrados entre Europa y África, y sólo con un estrecho canal de comunicación con el océano Atlántico a través del estrecho de Gibraltar. Éste se subdivide en dos subcuencas, el Mediterráneo oriental y el Mediterráneo occidental. El Mediterráneo es muy sensible y responde rápidamente a forzamientos atmosféricos $\mathrm{y} / \mathrm{o}$ a influencias antropogénicas (SCHROEDER et al. 2012). Además, presenta comportamientos atmosféricos propios y singulares, dada la protección y el aislamiento que le ofrece el relieve alrededor de su cuenca. El crecimiento demográfico, el cambio climático y la sobre explotación están ejerciendo una presión excepcional en el entorno mediterráneo y en sus ecosistemas y recursos. Además, se trata de una región donde los procesos oceánicos también tienen lugar, pero en unas dimensiones mucho menores que las que allí acontecen, como la formación de aguas profundas que contribuyen a mantener una célula de circulación termohalina de dimensiones iguales a las sub-cuencas, tal y como ocurre en el cinturón planetario a nivel oceánico (SCHROEDER et al. 2012). Esta realidad influye fuertemente en dotar a la región mediterránea de una marcada personalidad climática.

El concepto de fractal se utiliza para hacer referencia a objetos demasiado irregulares como para ser descritos según la geometría tradicional, pero que tienen la importante propiedad de ser invariantes por cambio de escala. La geometría fractal (MANDELBROT 1976) es una extensión de la geometría clásica y engloba la descripción, la clasificación y el análisis de subespacios geométricamente complicados. Generalmente, la estructura y organización de un conjunto fractal no hace posible especificar dónde, en lenguaje simple, se sitúa cada punto que lo compone. Por ello debe definirse alguna relación entre las diversas estructuras observadas en el mismo para varios niveles de resolución. Esta relación se formula cuantitativamente mediante el concepto de dimensión fractal, que describe el comportamiento escalar de las estructuras fractales.

Al igual que sucede con los objetos fractales, los procesos y sistemas invariantes por cambio de escala no poseen una escala que los caracterice. Teniendo esto en cuenta, un proceso fractal es aquel en el que el mismo proceso elemental tiene lugar a distintas escalas, es decir, en el que una parte reproduce el todo. Para el intervalo de escalas analizadas, los estadísticos del proceso siguen leyes potenciales caracterizadas por sus exponentes. La relación entre los estadísticos para las diferentes escalas consistirá simplemente en cocientes de escala. A modo de generalización, las dimensiones fractales, al contrario que las dimensiones corrientes o euclídeas que son siempre números enteros no negativos $(0$ para el caso de un punto, 1 para una recta, 2 para un plano, 3 para un espacio en tres dimensiones, etc.), pueden presentar un valor real no negativo. Para obtener una imagen más clara de esto, hay que suponer un punto que se desplaza sobre un plano describiendo un movimiento browniano, es decir, un movimiento aleatorio que tienda a completar o rellenar paulatinamente dicho plano. En un principio, su dimensión será 0 , pero en el mismo instante en el que empiece a describir dicho movimiento, su dimensión fractal pasará a ser 1, e inmediatamente 
después empezará a aumentar los decimales, pero sin llegar a dos, ya que no completará dicho plano.

Así, la geometría fractal y la dimensión fractal (como una dimensión no entera) son conocidas como una herramienta valiosa que permite describir la forma de los objetos, y que ha ganado una amplia utilización y aceptación en muchos campos de las ciencias naturales, incluyendo la Geografía, la Ecología o las nuevas tecnologías aplicadas a la información geográfica (GOODCHILD 1980; GOODCHILD \& MARK 1987; HASTINGS \& SUGIHARA 1994; PEITGEN et al. 1992). Los postulados de la geometría fractal han sido utilizados en la disciplina geográfica desde hace más de tres décadas. Fue introducida para medir la longitud de las líneas costeras de las Islas Británicas (MANDELBROT 1967), y posteriormente, su uso se ha dispersado de manera prolífica a una multitud de estudios en topografía. Una serie de algoritmos matemáticos están ahora disponibles para determinar la dimensión fractal para entidades lineales y de área. Un examen detallado de la bibliografía muestra que no todas las características topográficas son fractales en todas las escalas estudiadas. $\mathrm{Si}$ bien la naturaleza de algunos fenómenos multifractales geográficos ha sido explorada en gran profundidad, no queda completamente entendido por qué algunos terrenos o superficies están mejor modelados con la geometría fractal que otros.

El análisis fractal se ha utilizado con éxito para medir y caracterizar rasgos lineales irregulares como las costas, para describir y caracterizar las formas terrestres, y para regionalizar espacios estadísticamente según la forma de relieve. El análisis fractal también se puede utilizar para producir simulaciones de terreno con una dimensión conocida. El fracaso deriva del hecho de que no existe una relación de uno a uno entre los procesos geomorfológicos y las formas de relieve que se generan (GAO \& XIA 1996). La metodología de análisis fractal también se ha aplicado desde hace algunas décadas a los estudios de carácter climático, y es que algunas de sus variables (temperatura, precipitación, presión atmosférica, entre otras) tienen un comportamiento fractal, tanto espacial como temporalmente, hasta el punto que determinan la persistencia de estas variables y sus respectivas dependencias mutuas (REHMAN 2009). A partir de las series de datos de las tres grandes variables climáticas (temperatura, precipitación y presión atmosférica) y de las variabilidades de las mismas, mes a mes y entre temporadas, es posible definir cómo los modelos climáticos regionales no son capaces de elaborar una buena predicción del clima local, ya que únicamente trabajan con cantidades promediadas. Así aparecen hechos tan interesantes como que en regiones como la India, las precipitaciones durante el monzón del suroeste se ven afectadas por la variabilidad de la temperatura y la presión del invierno anterior. En esta misma línea, otros índices de predicción que incorporan los fractales son más fiables ya que consideran más dinámicas climáticas, con lo que se deduce que estos nuevos modelos mejoran los existentes en una escala regional (RANGARAJAN \& SANT 1997; RANGARAJAN \& SANT 2004).

Multitud de modelos se han desarrollado en Hidrología a partir de las propiedades fractales de la distribución temporal y espacial de la precipitación (ZHOU 2004; KHAN \& SIDDIQUI 2012). La utilidad de estos modelos de procesos hidrológicos de cuenca se incrementa considerablemente cuando pueden ser extrapolados a través de escalas espaciales y temporales. Este problema de la transferencia de escala, es decir, la descripción y la predicción de las características y los procesos a una escala diferente de aquella en la que se realizan las observaciones y mediciones, se ha convertido en el tema de mucha investigación actual en Hidrología y otras áreas afines. Efectivamente, este tipo de 
dinámicas se han identificado en estudios en la España peninsular a partir de series largas (noventa años) de precipitación acumulada anual, y su análisis revela que la distribución de esta variable se ajusta a una distribución fractal (OÑATE RUBALCABA 1997). Los valores obtenidos, con una media de 1,32 de dimensión fractal para todo el territorio, se encuentran en el mismo orden de magnitud que las dimensiones fractales obtenidas a partir de otros registros macrometeorológicos y paleoclimáticos (OÑATE RUBALCABA 1997).

En otras regiones del globo, como en la península de Shandong, en China, donde el problema de acceso a unos recursos hídricos cada vez más escasos es creciente al tratarse de una de las zonas de mayor y más rápido desarrollo del gigante asiático, el conocimiento de las tendencias de las precipitaciones se presenta como un tema crucial para el desarrollo futuro (REHMAN \& SIDDIQUI 2009; GAO \& HOU 2012). En la misma línea de los modelos multifractales, también se han llevado a cabo aplicaciones a estudios en la Península Ibérica, para el caso del observatorio de Córdoba, en el sur de España (DUNKERLEY 2008; GARCÍA-MARÍN et al. 2008). Sin embargo, se ha demostrado que las precipitaciones extremas se ajustan a modelos más complejos todavía que los multifractales, ya que se ven afectados por períodos limitantes, como duraciones muy cortas o períodos de retorno muy largos (LANGOUSIS et al. 2009; VENEZIANO \& FURCOLO 2002; VENEZIANO et al. 2006). En este tipo de estudios juega un papel determinante la resolución temporal con la que se trabaje, y es que el hecho de trabajar con datos de horarios, por una parte, y con datos diarios por otra, ya provoca cambios en los valores de las dimensiones fractales, también en parte por la influencia de la precipitación más característica de cada lugar (GARCÍA MARÍN 2007; LÓPEZ LAMBRAÑO 2012). Además, este método de análisis de la fractalidad permite la discriminación de mejores métodos de análisis para las frecuencias de la precipitación, coincidiendo con estudios mencionados anteriormente (GAO \& HOU 2012), llegando incluso a poder definir el régimen de precipitaciones de una región concreta (DUNKERLEY 2010; KUTIEL \& TRIGO 2014; REISER \& KUTIEL 2010).

Por todos estos factores, cobran especial relevancia los estudios de la variabilidad de la precipitación en la España peninsular, porque se trata no únicamente de una variable más del sistema climático, sino también de un recurso con enormes implicaciones en el aparato productivo, y porque, bajo esta escala de trabajo, son numerosos los factores que intervienen y, por tanto, modifican el reparto de las precipitaciones a nivel peninsular. No son escasos los estudios que se han llevado a cabo siguiendo esta temática, y es que los trabajos sobre la irregular distribución de la precipitación a escala peninsular han estado a la orden del día en las últimas décadas (ESTRELA et al. 2009; GRIMALT et al. 2006; MARTÍNVIDE et al. 2001; MARTÍN-VIDE 2004; MESEGUER-RUIZ \& MARTÍN-VIDE 2012; MESEGUER-RUIZ \& MARTÍNVIDE 2014; SARRICOLEA \& MARTÍNVIDE 2014; RODRÍGUEZ et al. 1999) mediante el estudio de diferentes índices estadísticos que son representativos de una mayor o menor irregularidad temporal de la precipitación a diversas escalas. Estos estudios se realizan mediante el análisis de cómo se reparten las precipitaciones a lo largo del año, ya sea año a año o de manera promediada, o también a partir de diferentes indicadores de irregularidad o disparidad temporal, como es el Índice de Concentración (CI). El objetivo del trabajo consiste en establecer la relación entre el CI y la dimensión fractal a nivel peninsular, para entender mejor cuál es el significado de este último índice. Para ello se llevará a cabo una correlación directa a través de la obtención del $r$ de Pearson y una correlación espacial. 


\section{MATERIALES Y MÉTODOS}

Se han utilizado las bases de datos de 20 observatorios de la red de estaciones automáticas de la Agencia Estatal de Meteorología (AEMet), de los cuales se han obtenido los datos de precipitación a resolución 10-minutal. Por otra parte, se ha seleccionado un período común para los observatorios en los cuales se obtenía una buena homogeneidad, considerando el período entre 1997 y 2010.

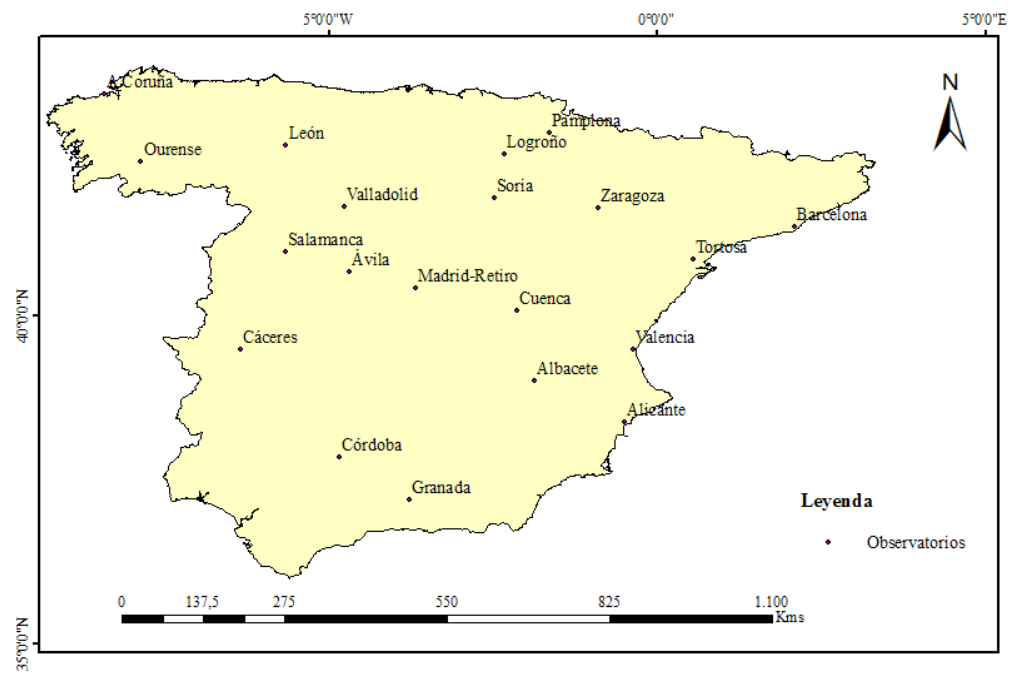

Fig. 1. Localización de los observatorios utilizados en la investigación.

Fig. 1. Localization of the used observatories in this research.

El cálculo de la dimensión fractal (D) se efectúa según el método box-counting, de la siguiente manera. A partir de registros pluviométricos a resolución 10-minutal, se considera el período de 10 minutos como el intervalo unitario de base para llevar a cabo el análisis. A continuación se han establecido períodos que contuvieran 1 , $2,3,6,12,18,24,36,48,72,144$ у 288 intervalos unitarios, es decir, períodos de 10,20 y 30 minutos, $1,2,3,4,6,8,12$, 24 y 48 horas respectivamente, y se ha contabilizado en cuántos de ellos se registra alguna cantidad de precipitación. Se define el valor de la dimensión fractal de la distribución temporal de la precipitación en base a la pendiente de la recta de regresión resultante de representar los pares de valores obtenidos a partir de los logaritmos naturales de 1 , extensión o longitud del intervalo, y de $\mathrm{N}$, número de intervalos con precipitación. En efecto, los logaritmos de esos pares de valores para cada observatorio se alinean con notable aproximación. La dimensión fractal (D) viene dada por $1+\alpha$, donde $\alpha$ es el valor absoluto de la pendiente de la recta de regresión (Fig. 2). 


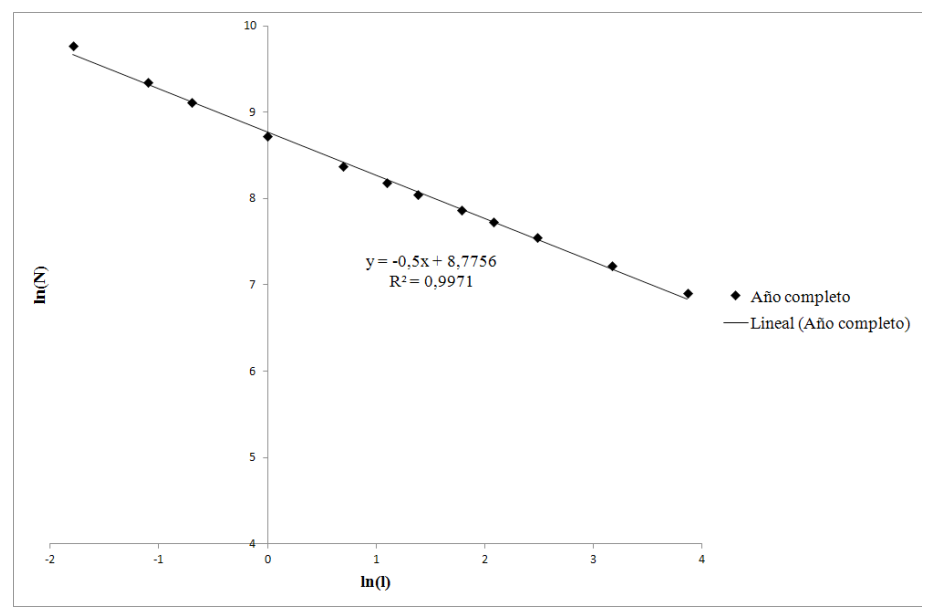

Fig. 2. Determinación de la Dimensión Fractal en Ávila.

Fig. 2. Determination of the Fractal Dimension in Ávila.

El Índice de Concentración (CI) se define como una aproximación al índice de Gini, representación numérica de las desigualdades mostradas por la curva de Lorenz (Fig. 3), que sirve para expresar el grado de concentración de una magnitud concreta en una porción de una población determinada. En este caso, el CI sirve para cuantificar la importancia de los días lluviosos respecto al total de lluvia acumulada en una serie temporal. Así, para determinar el impacto relativo de las diferentes clases de precipitación diaria, y especialmente para evaluar el paso de las mayores cantidades diarias recogidas respecto del total, este índice analiza los porcentajes acumulados de precipitación $Y$, al que contribuye el porcentaje acumulado de días $X$ en los cuales tuvo lugar (MARTÍNVIDE 2004).

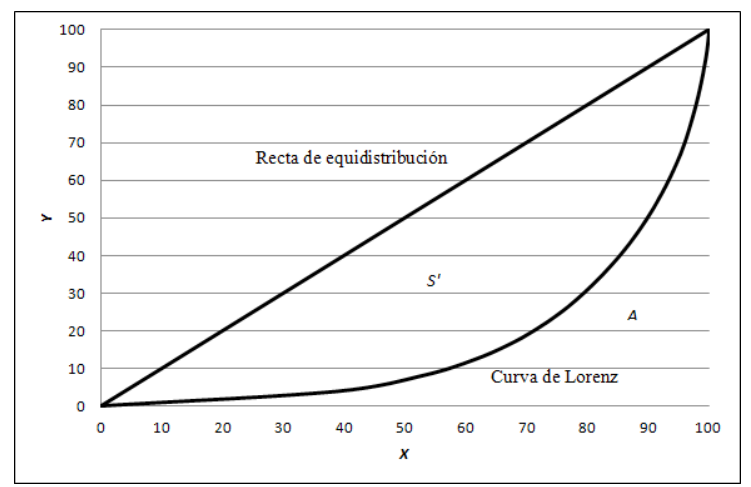

Fig. 3. Ejemplo de curva de concentración.

Fig. 3. Example of a concentration curve. 
Estos porcentajes se asocian a curvas exponenciales, del tipo:

$$
Y=a X \exp (b X)
$$

donde $a$ y $b$ son constantes.

Los valores de $a$ y $b$ se determinan de la siguiente manera:

$$
\begin{gathered}
\ln a=\frac{\sum X_{i}^{2} \sum \ln Y_{i}+\sum X_{i} \sum X_{i} \ln X_{i}-\sum X_{i}^{2} \sum \ln X_{i}-\sum X_{i} \sum X_{i} \ln Y_{i}}{N \sum X_{i}^{2}-\left(\sum X_{i}\right)^{2}} \\
b=\frac{N \sum X_{i} \ln Y_{i}+\sum X_{i} \sum \ln X_{i}-N \sum X_{i} \ln X_{i}-\sum X_{i} \sum \ln Y_{i}}{N \sum X_{i}^{2}-\left(\sum X_{i}\right)^{2}}
\end{gathered}
$$

Una vez que las constantes han sido determinadas, la integral definitiva de la curva exponencial entre 0 y 100 es el área $A$ bajo la curva:

$$
A=\int_{0}^{100} a X e^{b X} d X
$$

El área $S$ ' comprimida por la curva, la línea de equidistribución y $X=100$ es la diferencia entre 5000 y el valor de la ecuación del valor de $A$ :

$$
S^{\prime}=5000-A
$$

A partir de este valor, se define el índice de concentración de la precipitación diaria:

$$
C I=\frac{2 S^{\prime}}{10000}=\frac{S^{\prime}}{5000}
$$

Se debe notar que el valor del CI es la fracción de $S$ ' y la superficie del área del triángulo inferior delimitado por la recta de equidistribución.

Los resultados de D y de CI que se obtengan para cada observatorio se correlacionarán directamente para obtener el coeficiente de correlación de Pearson ( $\mathrm{r}$ de Pearson). Posteriormente, se llevarán estos resultados a un mapa, donde se interpolará a partir del método kriging para obtener unos valores de ambos índices para todo el territorio, resultando dos capas raster. Posteriormente, se procede a correlacionar ambas capas para obtener el valor de la correlación espacial entre D y CI.

\section{RESULTADOS}

La aplicación de la metodología descrita anteriormente proporciona los valores de D y del CI presentados en la Tabla 1, obteniéndose los correspondientes datos

\begin{tabular}{|c|c|c|}
\hline Observatorio & D & CI \\
\hline Albacete & 1,4941 & 0,59 \\
\hline Alicante & 1,4710 & 0,68 \\
\hline Ávila & 1,5000 & 0,60 \\
\hline Barcelona & 1,5071 & 0,65 \\
\hline Cáceres & 1,5464 & 0,57 \\
\hline Córdoba & 1,5605 & 0,58 \\
\hline Cuenca & 1,5468 & 0,56 \\
\hline Granada & 1,5941 & 0,56 \\
\hline La Coruña & 1,5629 & 0,56 \\
\hline León & 1,5578 & 0,57 \\
\hline Logroño & 1,4961 & 0,59 \\
\hline Ourense & 1,5704 & 0,55 \\
\hline Pamplona & 1,5487 & 0,58 \\
\hline Madrid-Retiro & 1,5432 & 0,60 \\
\hline Salamanca & 1,5075 & 0,57 \\
\hline Soria & 1,5190 & 0,56 \\
\hline Tortosa & 1,5167 & 0,69 \\
\hline Valencia & 1,5258 & 0,70 \\
\hline Valladolid & 1,5261 & 0,58 \\
\hline Zaragoza & 1,5154 & 0,62 \\
\hline
\end{tabular}
para los 20 observatorios utilizados.

\section{Tabla 1: Valores de D y CI de los DIFERENTES OBSERVATORIOS.}

Table 1: Values of D and CI of the DIFFERENT OBSERVATORIES.

Los valores extremos obtenidos para la dimensión fractal son aquellos de Alicante $(1,4710)$ y Granada (1,5941), mientras 
que los extremos obtenidos para el Índice de Concentración son aquellos que se dan en Ourense $(0,55)$ y en Valencia $(0,70)$. Se representan en la Figura 4 los valores interpolados para toda el área de estudio del
CI mediante la metodología del kriging. Se ha aplicado la misma metodología para la distribución espacial de la dimensión fractal (Fig. 5).

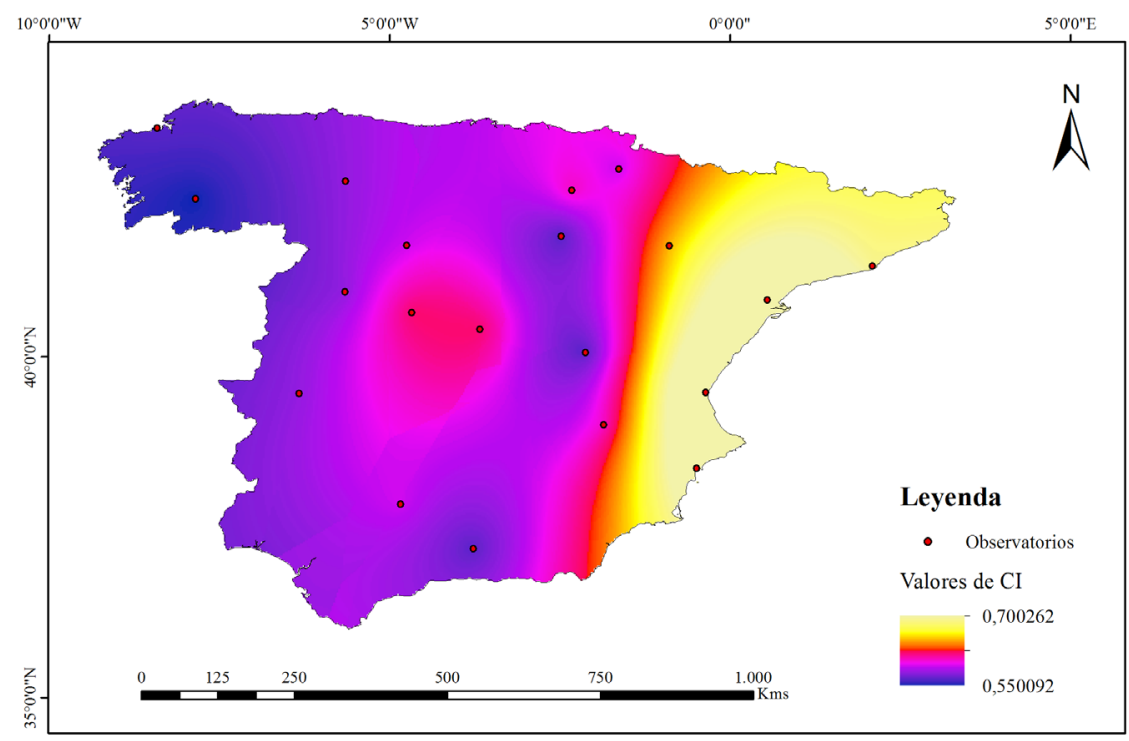

Fig. 4. Distribución espacial del Î́ndice de Concentración en la Península Ibérica.

Fig. 4. Spatial distribution of the Concentration Index in the Iberian Peninsula.

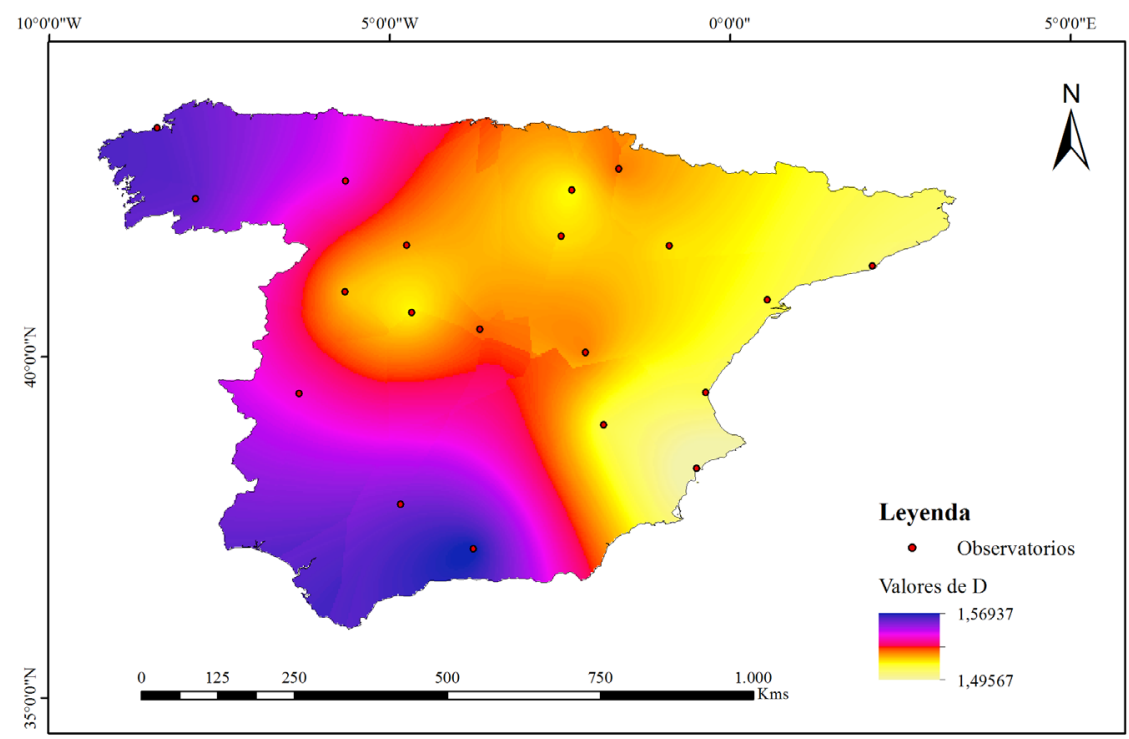

Fig. 5. Distribución espacial de la Dimensión Fractal en la Península Ibérica.

Fig. 5. Spatial distribution of the Fractal Dimension in the Iberian Peninsula. 
Es apreciable cómo los valores más elevados del CI se concentran en el litoral mediterráneo, decreciendo hacia el oeste, mientras que la dimensión fractal concentra sus valores más bajos en la costa oriental, y los valores aumentan hacia el norte y hacia el sur. Al correlacionar ambas variables de manera directa, se obtiene un valor de la $\mathrm{r}$ de Pearson de -0,55 (Fig. 6), con un nivel de confianza superior al $99 \%$ ( $p$-value de 0,008$)$.

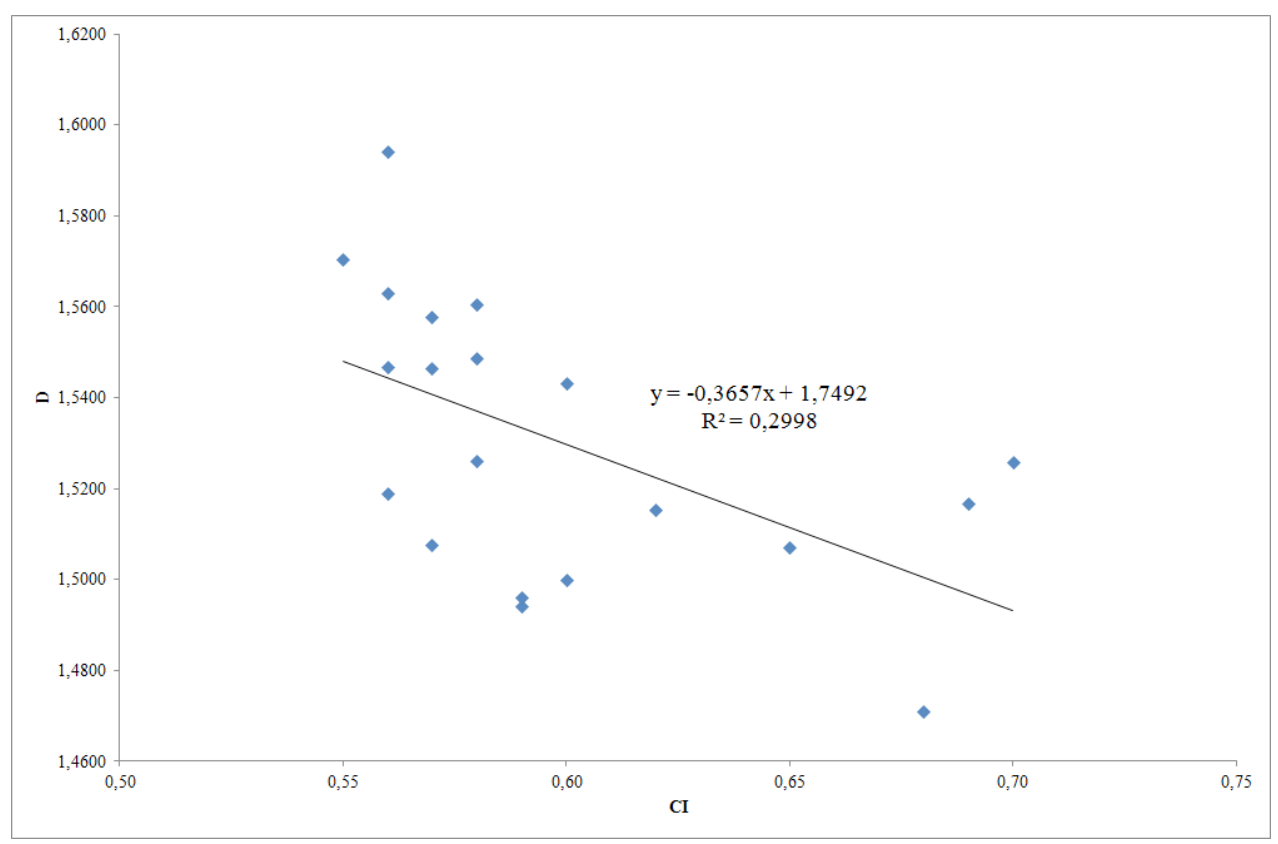

Fig. 6. Relación lineal entre los valores de CI y D.

Fig. 6. Linear relationship between the values of CI and D.

Se observa cómo los valores de D y CI se ajustan con una buena precisión. Sin embargo, alrealizarunanálisis geoestadístico y llevar a cabo una correlación espacial, el índice de correlación es de $-0,72$, con un nivel de confianza superior al $99 \%$. De esto se deduce que el Índice de Concentración y la dimensión fractal de la precipitación estén estrechamente relacionados.

\section{DISCUSIÓN Y CONCLUSIONES}

La dimensión fractal (D) y el Índice de Concentración (MARTÍN-VIDE 2004) guardan una buena correlación negativa $(-0,55)$, significativa al $99 \%$, siendo aun de mayor magnitud si se lleva a cabo a nivel espacial (-0,72, también en el máximo nivel de confianza). El CI representa el peso de los días más lluviosos respecto al total de días lluviosos de una serie. Por lo tanto, afirmar que guarda una correlación negativa con la dimensión fractal implica que a mayor dimensión fractal, menor CI, y por lo tanto, la precipitación se encuentra más repartida en varios días lluviosos en vez que unos pocos. Esto significa que a mayor valor de la dimensión fractal, el peso específico de los días con mayor precipitación acumulada es menor que en las regiones con menor valor de dimensión fractal. Esto es así ya que los mayores valores del CI en la Península Ibérica se dan en Levante, donde es posible que se recojan más de 200 litros en menos 
de 24 horas, y también es donde los valores de D son más bajos. Así, a mayor dimensión fractal, la precipitación queda mejor repartida a lo largo de la serie. Valores del CI más bajos, como los que se dan en las áreas más septentrionales de la zona de estudio (vertiente atlántica), evidencian un gradiente longitudinal en el comportamiento espacial del CI, como también ocurre en el caso del comportamiento espacial de $\mathrm{D}$, aunque en este caso se aprecia una mayor influencia de la continentalidad, por lo que puede intuirse que la distancia al mar también es un factor que interviene en la distribución espacial de los valores de D. Es difícil comparar estos resultados con otros estudios similares, ya que no se ha aplicado esta metodología con esta resolución temporal de los datos en ninguna otra parte, siendo la mayor resolución la diaria.

El Índice de Concentración y la dimensión fractal guardan una buena correlación negativa y significativa entre ellos. Altos valores de $\mathrm{D}$ están vinculados a una escasa concentración diaria de los montos de precipitación acumulados en 24 horas. Estos valores más elevados se dan en la vertiente atlántica de la España peninsular, donde la lluvia se vincula mayormente a la que procede de las borrascas atlánticas, de naturaleza estratiforme y que suele tener una duración mayor que la lluvia de origen convectivo, que tienen más peso en los totales recogidos en los observatorios de la España mediterránea, donde se dan los valores de CI más elevados y los valores de $\mathrm{D}$ más bajos.

\section{AGRADECIMIENTOS}

Esta investigación se inserta dentro del proyecto PRECABAL (CGL201129263-C02-01) del Ministerio de Ciencia e Innovación del Gobierno de España, y en el marco de las investigaciones del Grupo de Climatología de la Universidad de Barcelona (2014SGR300) y de su Institut de
l'Aigua. También se agradece al proyecto FONDECYT de Iniciación 11130629 del Gobierno de Chile.

\section{REFERENCIAS}

DUNKERLEY, D.L., 2008. Rain event properties in nature and in rainfall simulation experiments: a comparative review with recommendations for increasingly systematic study and reporting. Hydrological Processes, 22: 4415-4435.

DUNKERLEY, D.L., 2010. How do the rain rates of sub-events intervals such as the maximum 5- and 15-min rates $\left(\mathrm{I}_{5}\right.$ or $\mathrm{I}_{30}$ ) relate to the properties of the enclosing rainfall event?. Hydrological Processes, 24: 2425-2439.

ESTRELA, M.J., J. MIRÓ, F. PASTOR \& M. MILLÁN, 2009. Tendencias en la precipitación, por distintos inputs, en el sector central de la Península Ibérica (1958-2008). Investigaciones Geográficas, 49: 159-172.

GAO, J. \& Z. XIA, 1996. Fractals in physical geography. Progress in Physical Geography, 20(2): 178-191.

GAO, M. \& X. HOU, 2012. Trends and Multifractals Analyses of Precipitation Data from Shandong Peninsula, China. American Journal of Environmental Sciences, 8(3): 271-279.

GARCÍA MARÍN, A.P., 2007. Análisis multifractal de series de datos pluviométricos en Andalucía. Tesis doctoral, Departamento de Ingeniería Rural, Escuela Técnica Superior de Ingenieros Agrónomos y Montes, Universidad de Córdoba.

\section{GARCÍA-MARÍN, A.P., F.J. JIMÉNEZ-} HORNERO \& J.L. AYUSO-MUÑOZ, 2008. Universal multifractal description of an hourly rainfall time series from a location in southern Spain. Atmósfera, 21(4): 347-355. 
GOODCHILD, M.F., 1980. Fractals and the accuracy of geographical measures. Mathematical Geology, 12(2): 85-98.

GOODCHILD, M.F. \& D.M. MARK, 1987. The Fractal Nature of Geographic Phenomena. Annals of the Association of American Geographers, 77(2): 265-278.

GRIMALT, M., M. LAITA, J. ROSSELLÓ, J. CALDENTEY \& J.M. ARROM, 2006. Distribución espacial y temporal de las precipitaciones intensas en Mallorca. En CUADRAT PRATS, J.M., SAZ SÁNCHEZ, M.A., VICENTE SERRANO, S.M., LANJERI, S., DE LUIS ARRILLAGA, M. \& J.C. GONZÁLEZHIDALGO (Eds.), Clima, Sociedad y Medio Ambiente. Publicaciones de la Asociación Española de Climatología, Zaragoza: 411-420.

HASTINGS, H.M. \& G. SUGIHARA, 1994. Fractals: A User's Guide for the Natural Sciences. Oxford University Press, Oxford.

KHAN, M.S. \& T.A. SIDDIQUI, 2012. Estimation of fractal dimension of a noisy time series. International Journal of Computer Applications, 45(10): 1-6.

KUTIEL, H. \& R.M. TRIGO, 2014. The rainfall regime in Lisbon in the last 150 years. Theoretical and Applied Climatology, DOI 10.1007/s00704-013-1066-y.

LANGOUSIS, A., D. VENEZIANO, P. FURCOLO \& C. LEPORE, 2009. Multifractal rainfall extremes: Theoretical analysis and practical estimation. Chaos, Solitons and Fractals, 39: 1182-1194.

LÓPEZ LAMBRAÑO, A.A., 2012. Análisis multifractal y modelación de la precipitación. Tesis doctoral, Facultad de Ingeniería, Universidad Autónoma de Querétaro.
MANDELBROT, B.B., 1967. How long is the coast of Britain? Statistical self-similarity and fractional dimension. Science, 156: 636-638.

MANDELBROT, B.B., 1976. The Fractal Geometry of Nature. W.H. Freeman and Company, New York.

MARTÍN-VIDE, J., M. PROHOM, M. BOHIGAS, J.C. PEÑA, P. ESTEBAN \& D. MONTSERRAT, 2001. Índices de irregularidad temporal y dimensión fractal de la precipitación anual en España. En PÉREZ-CUEVA, A.J., LÓPEZ BAEZA, E. \& J. TAMAYO CARMONA (Eds.), El Tiempo del Clima. Publicaciones de la Asociación Española de Climatología, Valencia, 157-166.

MARTÍN-VIDE, J., 2004. Spatial distribution of a daily precipitation concentration index in Peninsular Spain. International Journal of Climatology, 24: 959-971.

MESEGUER-RUIZ, O. \& J. MARTÍNVIDE, 2012. Análisis de la fractalidad temporal de la precipitación en Cataluña durante 2010 y 2011. En RODRÍGUEZ PUEBLA, C., CEBALLOS BARBANCHO, A., GONZÁLEZ REVIRIEGO, N., MORÁN TEJEDA, E. y A. HERNÁNDEZ ENCINAS (Eds.), Cambio climático. Extremos e impactos. Publicaciones de la Asociación Española de Climatología, Salamanca: 539-547.

MESEGUER-RUIZ, O. \& J. MARTÍNVIDE, 2014. Análisis de la fractalidad temporal de la precipitación en Cataluña, España (2010). Investigaciones Geográficas, 47: 41-52.

PEITGEN, H.O., H. JÜRGENS \& D. SAUPE, 1992. Chaos and Fractals: New Frontiers of Science. Springer, New York. 
OÑATE RUBALCABA, J.J., 1997. Fractal Analysis of Climatic Data: Annual Precipitation Records in Spain. Theoretical and Applied Climatology, 56: 83-87.

RANGARAJAN, G. \& D.A. SANT, 1997. A climate predictability index and its applications. Geophysical Research Letters, 24: 1239-1242.

RANGARAJAN, G. \& D.A. SANT, 2004. Fractal dimensional analysis of Indian climatic dynamics. Chaos, Solitons and Fractals, 19: 285-291.

REHMAN, S., 2009. Study of Saudi Arabian climatic conditions using Hurst exponent and climatic predictability index. Chaos, Solitons and Fractals, 39: 499-509.

REISER, H. \& H. KUTIEL, 2010. Rainfall uncertainty in the Mediterranean: Intraseasonal rainfall distribution. Theoretical and Applied Climatology, 100: 105-121.

RODRÍGUEZ, R., M.C. LLASAT \& D. WHEELER, 1999. Analysis of the Barcelona precipitation series 1850-1991. International Journal of Climatology, 19: 787-801.

SARRICOLEA, P. \& J. MARTÍN-VIDE, 2014. Spatial analysis of rainfall daily trends and concentration in Chile. Investigaciones Geográficas, 47: 53-66.
SCHROEDER, K., J. GARCÍALAFUENTE, S.A. JOSEY, V. ARTALE, B. BUONGIORNO NARDELLI, A. CARRILlO, M. GAČIĆ, G.P. GASPARINI, M. HERRMAN, P. LIONELLO, W. LUDWIG, C. MILLOT, E. ÖZSOKY, G. PISACANE, J.C. SÁNCHEZ-GARRIDO, G. SANNINO, R. SANTOLERI, S. SOMOT, M. STRUGLIA, E. STANEV, I. TAUPIERLETAGE, M.N. TSIMPLIS, M. VARGAS-YÁÑEZ, V. ZERVAKIS \& G. ZODIATIS, 2012. Circulation of the Mediterranean Sea and it's Variability, en PILONELLO, P. (Ed.): The Climate of the Mediterranean Region. From the Past to the Future, Elsevier Insights, London: 187-256.

VENEZIANO, D. \& P. FURCOLO, 2002. Multifractality of rainfall and scaling of intensity-duration-frequency curves. Water Resources Research, 38(12), 1306, doi: 10.1029/2001WR000372.

VENEZIANO, D., A. LANGOUSIS \& P. FURCOLO, 2006. Multifractality and rainfall extremes: A review. Water Resources Research, 42, W06D15, doi: 10.1029/2005WR004716.

ZHOU, X., 2004. Fractal and Multifractal Analysis of Runoff Time Series and Stream Networks in Agricultural Watersheds. Tesis doctoral, Virginia Polytechnic Institute and State University. 